\title{
GroupTracker: Video Tracking System for Multiple Animals under Severe Occlusion
}

\author{
Tsukasa Fukunaga ${ }^{\mathrm{a}, \mathrm{b}, *}$, Shoko Kubota ${ }^{\mathrm{c}}$, Shoji Oda ${ }^{\mathrm{c}}$, Wataru Iwasaki ${ }^{\mathrm{a}, \mathrm{b}, \mathrm{d}, * *}$ \\ ${ }^{a}$ Department of Computational Biology, Graduate School of Frontier Sciences, The \\ University of Tokyo, 27r7-8568, Chiba, Japan \\ ${ }^{b}$ Atmosphere and Ocean Research Institute, The University of Tokyo, 277-8564, Chiba, \\ Japan \\ ${ }^{c}$ Department of Integrated Biosciences, Graduate School of Frontier Sciences, The \\ University of Tokyo, 277-8562, Chiba, Japan \\ ${ }^{d}$ Department of Biological Sciences, Graduate School of Science, The University of \\ Tokyo, 113-0032, Tokyo, Japan
}

\begin{abstract}
Quantitative analysis of behaviors shown by interacting multiple animals can provide a key for revealing high-order functions of their nervous systems. To resolve these complex behaviors, a video tracking system that preserves individual identity even under severe overlap in positions, i.e., occlusion, is needed. We developed GroupTracker, a multiple animal tracking system that accurately tracks individuals even under severe occlusion. As maximum likelihood estimation of Gaussian mixture model whose components can severely overlap is theoretically an ill-posed problem, we devised an ExpectationMaximization scheme with additional constraints on the eigenvalues of the covariance matrix of the mixture components. Our system was shown to accurately track multiple medaka (Oryzias latipes) which freely swim around in three dimensions and frequently overlap each other. As an accurate multiple animal tracking system, GroupTracker will contribute to revealing unexplored structures and patterns behind animal interactions. The Java source code of GroupTracker is available at https://sites.google.com/site/fukunagats $\mathrm{u} /$ software/group-tracker.
\end{abstract}

\footnotetext{
*Corresponding Author. TEL: +81-3-5841-3046. E-mail address: fukunaga@cb.k.utokyo.ac.jp

** Corresponding Author. TEL: +81-3-5841-3047. E-mail address: iwasaki@bs.s.utokyo.ac.jp
} 
Keywords: Bioimage informatics, Computational ethology, Animal tracking

\section{Introduction}

Animals display a wide range of complex behaviors. Among them, behaviors shown by interacting multiple animals are particularly interesting. Video analyses of those behaviors can reveal high-order functions of their nervous systems ([31]; [24]; [26]). Furthermore, advancements in quantitative behavioral measurements have broad applicability in studies of neurological disease, by enabling systematic isolation of mutant strains of model organisms and objective analyses of their phenotypes on inter-individual interactions ([28]).

In this study, we focus on tracking of multiple animal individuals that have similar appearances. While this is a general framework for analyzing a group of individuals of the same species, the issue of identity preservation during tracking is not trivial. In particular, this problem becomes serious when individuals severely overlap each other in image frames, i.e., under severe occlusion. Such occlusion occurs not only under typical experimental settings but also during interesting inter-individual behaviors such as courtships ([27]). Thus, most studies so far required laborious manual annotations of identities and positions of individuals, and the ability to perform large-scale systematic analyses is greatly inhibited.

To date, a number of video tracking systems have been developed, for example, for nematodes ([3]; [30]; [32]), mice ([10]; [13]; [25]), fruit flies ([9]; [6]), ants ([18]), and fish ([17]; [21]), although none focused on solving the severe occlusion problem. Some developed methods for single individual tracking (e.g., [3]), dealt with cases with few occlusions (e.g., [11]), or physically prohibited occlusions (e.g., [21]). This was partly because frequently adopted methods, such as mathematical morphology ([17]) and watershed algorithm ([13]), had difficulties in separating severely overlapping individuals.

Recently, the Gaussian mixture model has been adopted by several multiple animal tracking methods, where animal individuals are represented by components of a Gaussian mixture $([9] ;[6]$; [33]; [25]). Through this approach, latent variables such as true positions of individuals are explicitly represented. The associated probability models and numerical methods are also well-established. Although a Gaussian distribution cannot represent, for example, bending shapes of a nematode, it has been successfully applied to 
many animals such as mice and fruit flies ([9]; [25]). Nevertheless, methods adopting the Gaussian mixture model also suffer from the severe occlusion problem, because the maximum likelihood estimation of the Gaussian mixture model is theoretically an ill-posed problem under the condition where multiple components can overlap ([5]).

In this study, we developed a Gaussian mixture model-based, multiple animal tracking system that accurately tracks individuals even under severe occlusion. The key idea was the introduction of constraints to the eigenvalues of the covariance matrices of the Gaussian mixture components, by taking advantage of the fact that the size of each individual usually remains almost constant during a video sequence. We developed algorithm that effectively estimates the Gaussian mixture parameters under these additional constraints, and implemented a publicly available software tool named 'GroupTracker' (GROUP: Gaussian Reinterpretation of OcclUsion Problem).

\section{Material and Methods}

\subsection{Video Sequence Dataset}

Medaka fish (Oryzias latipes) was selected for demonstrative purposes in this study. As fish swim around in three dimensions and frequently overlap each other, they are suitable for evaluating multiple animal tracking system under occlusion conditions. It should be noted that, partly because of these characteristics, tracking systems for fish are underdeveloped compared with those for other organisms ([12]). Furthermore, medaka fish has been used as a model organism in many fields of animal sciences. It shows various interesting behaviors that involve inter-individual interactions such as schooling and aggressive behaviors ([20]), while rich resources are available for its neurobiology and genomics ([1]; [16]).

Five ten-minute video sequences that recorded one, two, four, eight, and sixteen individuals were prepared. Medaka fish (Hd-rR strain) were hatched and bred in laboratory aquariums. In each case, equal numbers of female and male individuals (one female in the case of one individual) at six months of age (adult, body lengths $\approx 3 \mathrm{~cm}$ ) were transferred to a white, opaque, cylindrical ring-shaped, plastic water tank (outer radius $=46 \mathrm{~cm}$, inner radius $=24 \mathrm{~cm}$, depth $\approx 4 \mathrm{~cm}$, water temperature $=26^{\circ} \mathrm{C}$; Fig. $\left.1 \mathrm{~A}\right)$. This shape of the tank enhanced the schooling behavior of medaka. A white polarized LED lamp $(10.7 \mathrm{~cm} \times 22.5 \mathrm{~cm})$ located above the tank was used as the light source during video recording (Supplementary Fig. 1). A high-definition 
digital video recorder (HDR-HC9 Sony Corp., Japan) was set approximately $140 \mathrm{~cm}$ above the water surface. A polarizing filter (VF-37CPKS, Sony Corp., Japan) was used to reduce light reflection. Videos were recorded in elevenminute sequences during daytime (from $2 \mathrm{pm}$ to $5 \mathrm{pm}$ ) using default video settings. Blackout curtain was set up surrounding the entire apparatus to prevent external (human) interference. Final Cut Pro (Apple Inc., U.S.A.) was used to convert the videos into the Motion JPEG format (frames per second $=30$, resolution of the image frames $=872 \times 480$ ). The first oneminute segment was deleted from each video sequence.

A

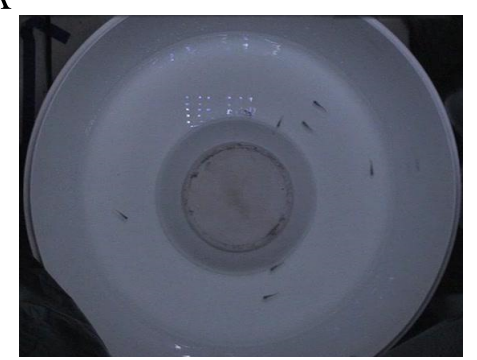

B

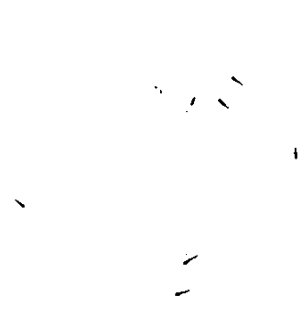

$\mathrm{C}$

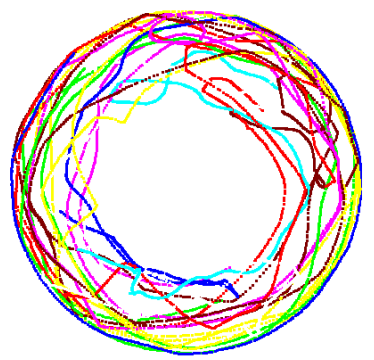

Figure 1: (A) An image frame in a video sequence that contained eight individual medaka fish. (B) The same image frame, after the preprocessing step. (C) Tracks of eight individuals from a one-minute video segment. Colors represent different individuals.

\subsection{Method Overview}

The method consists of three major steps: preprocessing, tracking, and post-processing. At the preprocessing step, objects outside of the movable areas (i.e., outside of the water boundary in case of fish) are removed and pixels composing the animal shapes are extracted from every image frame using conventional image-processing methods ([14]) (Fig. 2A). Then, the tracking step determines the precise position of each individual by fitting the Gaussian mixture model to the preprocessed image frames (Fig. 2B). The post-processing step consists of three minor steps: identity-swapping alert, identity-swapping correction, and head-direction determination. At the identity-swapping alert step, the system alerts the user to image frames that may contain identity-swapping errors. The identity-swapping correction step then automatically correct a portion of these errors. Finally, at the headdirection determination step, the direction of the head of each individual is determined in each image frame. 


\subsection{Preprocessing Step}

At this step, first, every image frame in the video sequence is converted to 8-bit grayscale (into the 0-255 range from dark to bright by the NTSC conversion) and, to remove light reflection, any values higher than the threshold value of 100 is set to this value. Next, dynamic threshold binarization and statistical background subtraction are conducted to select pixels that likely constitute animal shapes. The former technique selects every pixel whose brightness value is lower than a dynamic threshold that is the average brightness value of the surrounding pixels $(5 \times 5$ square pixels $)$ plus or minus a user-defined value. Because medaka's body were darker than the surrounding environment, the user-defined value was set to -5 . The latter technique selects every pixel whose brightness value is lower than a static threshold calculated as follows. Thirty image frames are collected at even intervals from the entire video sequence and, for each pixel coordinates, the mean $\mu$ and variance $\sigma$ of the brightness values are calculated. The static threshold is then set to $\mu-2 \sigma$. Common pixels selected by both techniques are obtained and a median filter is applied to remove noises. Finally, the remaining pixel set is passed on to the tracking step.
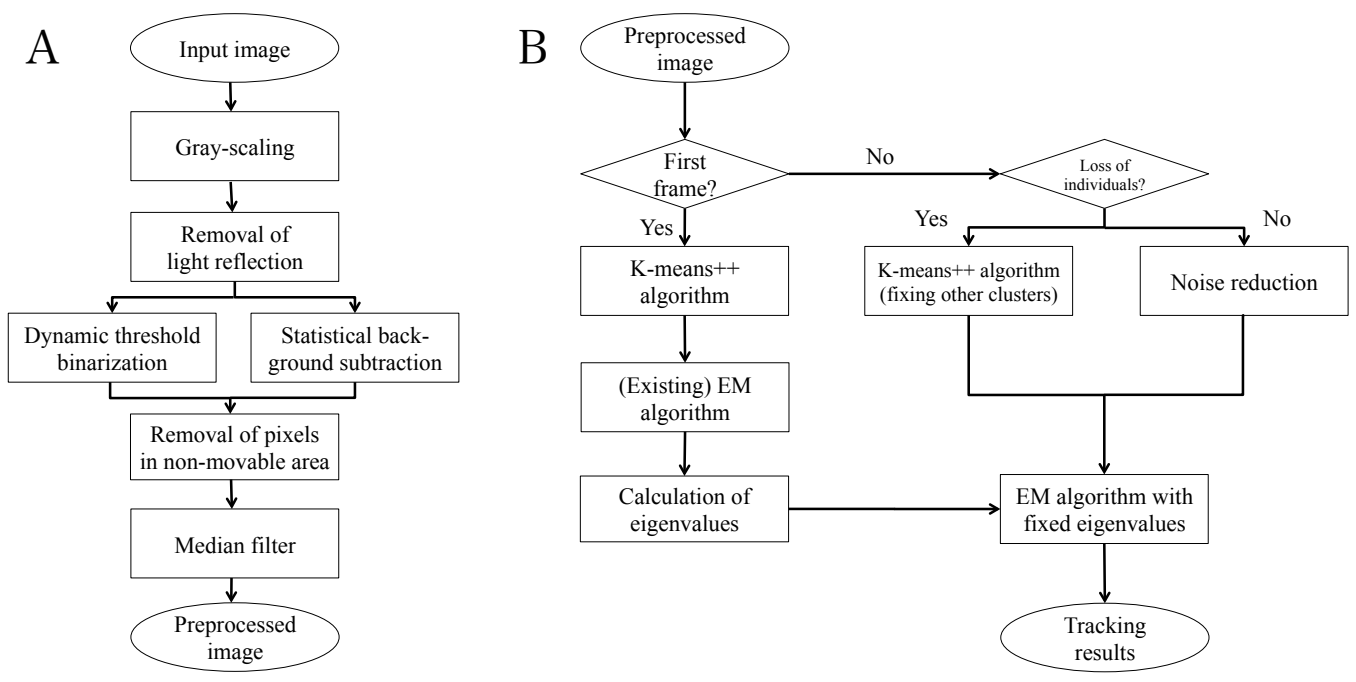

Figure 2: Overviews of the (A) preprocessing step and (B) tracking step. 


\subsection{Tracking Step}

At this step, the two-dimensional Gaussian mixture model is applied to the preprocessed images (Figs. 3A and 3B) using the same number of mixture components as that of animal individuals. Hence, the mean value and covariance matrix of each component represent the position and shape of each individual, respectively.

First, the system processes the first image frame. K-means++ algorithm ([19]; [2]) is applied to divide the pixels identified during the preprocessing step into $K$ clusters, where $K$ is the number of individuals. Because Kmeans ++ algorithm can converge to local optima, the clustering process is repeated $R=100$ times and the result with the smallest K-distance calculated as follows is chosen.

$$
\text { K-distance }=\sum_{k=1}^{K} \sum_{\mathbf{x} \in C_{k}} \frac{1}{\left|C_{k}\right|}\left(d\left(\mathbf{x}, \mathbf{c}_{\mathbf{k}}\right)\right)^{2}
$$

where $\mathbf{x}$ is a pixel coordinate, $C_{k}$ is a cluster, $\mathbf{c}_{\mathbf{k}}$ is the coordinate of its centroid, and $d(\cdot, \cdot)$ is the Euclidean distance. Then, the mean value $\mu_{k}$ and the covariance matrix $\boldsymbol{\Sigma}_{k}$ of each mixture component are set to $\mathbf{c}_{\mathbf{k}}$ and K-distance $\times 0.1 \times \mathbf{I}$, where $\mathbf{I}$ is the identity matrix, respectively. The mixture ratio of each component $\pi_{k}$ is set to $1 / K$.

Then, for each successive image frame, the parameters of the Gaussian mixture distributions are estimated by the Expectation-Maximization (EM) algorithm ([5]) using the parameter estimate of the previous frame as the initial values. This relies on an assumption that the position and shape of an individual do not change abruptly between adjacent frames, which is generally true when the number of frames per unit time is sufficiently large. It should be noted that this approach naturally preserves the identities of individuals in most cases.

In its original formulation, the EM algorithm described is as follows ([5]). The log-likelihood function is defined as:

$$
\ln p(\mathbf{X} \mid \boldsymbol{\pi}, \boldsymbol{\mu}, \boldsymbol{\Sigma})=\sum_{n=1}^{N} \ln \left\{\sum_{k=1}^{K} \pi_{k} \mathcal{N}\left(\mathbf{x}_{n} \mid \boldsymbol{\mu}_{k}, \boldsymbol{\Sigma}_{k}\right)\right\}
$$

where $N$ is the number of pixels determined during the preprocessing step of each image frame and $\mathcal{N}$ is the Gaussian probability density function. The E step calculates $\gamma\left(z_{n k}\right)=\frac{\pi_{k} \mathcal{N}\left(\mathbf{x}_{n} \mid \boldsymbol{\mu}_{k}, \boldsymbol{\Sigma}_{k}\right)}{\sum_{l} \pi_{l} \mathcal{N}\left(\mathbf{x}_{n} \mid \boldsymbol{\mu}_{l}, \boldsymbol{\Sigma}_{l}\right)}$, where $z_{n k}$ indicates whether $\mathbf{x}_{n}$ 
belongs to the mixture component $k$ and $\gamma\left(z_{n k}\right)$ represents 'responsibility' that the mixture component $k$ explains the observation $\mathbf{x}_{n}$. Then, the $\mathrm{M}$ step updates the parameters using $\gamma\left(z_{n k}\right)$. The $\mathrm{E}$ and $\mathrm{M}$ steps are repeated until the likelihood function converges to a local maximum.

Nevertheless, this EM algorithm could not be applied to the current problem because the maximum likelihood estimation of the Gaussian mixture model is intrinsically an ill-posed problem if any two components can severely overlap ([5]) (Fig. 3C). In this case, a Gaussian mixture component can collapse to a single pixel $\mathbf{x}$ and the likelihood function can contain the term $\mathcal{N}(\mathbf{x} \mid \mathbf{x}, \boldsymbol{\Sigma})=\left(2 \pi|\boldsymbol{\Sigma}|^{\frac{1}{2}}\right)^{-1}$, which diverges to infinity as $|\boldsymbol{\Sigma}| \rightarrow 0$.

Therefore, we developed a novel algorithm that overcomes this limitation. The key idea was to fix the eigenvalues of $\boldsymbol{\Sigma}_{k}$ since they represent the sizes of the individuals, which can be considered constant during a video sequence (Fig. 3D). If the eigenvalues are fixed, a Gaussian mixture component cannot collapse to a single pixel and $|\boldsymbol{\Sigma}|$ cannot approach 0 . First, the original EM algorithm described above is applied to the first image frame and the eigenvalues of $\boldsymbol{\Sigma}_{k}$ are calculated. This requires that all animal individuals do not overlap in the first frame, though it is trivial to choose any frame that fulfills this condition in a video sequence. Then, the adapted EM algorithm that maximizes the likelihood function while fixing the eigenvalues is applied to the first and subsequent frames, using the eigenvalues calculated above as input. Note that the likelihood function does not change even if the eigenvalues are fixed; in other words, only the $M$ step needs to be revised. Since the covariance matrix of a Gaussian distribution is a real symmetric matrix, we can choose the eigenvectors that form an orthonormal set ([5]). Given eigenvalues $\lambda_{i k}$ and eigenvectors $\mathbf{u}_{i k}$, the covariance matrix is written by

$$
\begin{aligned}
\boldsymbol{\Sigma}_{k} & =\lambda_{1 k} \mathbf{u}_{1 k} \mathbf{u}_{1 k}^{\mathrm{T}}+\lambda_{2 k} \mathbf{u}_{2 k} \mathbf{u}_{2 k}^{\mathrm{T}} \\
& =\left(\begin{array}{cc}
\lambda_{1 k} \cos ^{2} \theta_{k}+\lambda_{2 k} \sin ^{2} \theta_{k} & \left(\lambda_{1 k}-\lambda_{2 k}\right) \sin \theta_{k} \cos \theta_{k} \\
\left(\lambda_{1 k}-\lambda_{2 k}\right) \sin \theta_{k} \cos \theta_{k} & \lambda_{1 k} \sin ^{2} \theta_{k}+\lambda_{2 k} \cos ^{2} \theta_{k}
\end{array}\right)
\end{aligned}
$$

Note that we can set $\mathbf{u}_{1 k}$ to $\left(\cos \theta_{k}, \sin \theta_{k}\right)^{\mathrm{T}}$ and $\mathbf{u}_{2 k}$ to $\left(-\sin \theta_{k}, \cos \theta_{k}\right)^{\mathrm{T}}$ $\left(0 \leq \theta_{k}<\pi\right)$, where $\theta_{k} \in[0, \pi)$ is the angle of the major axis of the Gaussian component.

The log-likelihood function can also be represented by using $\theta_{k}, \lambda_{1 k}$, and $\lambda_{2 k}$. By calculating its partial derivatives with respect to $\theta_{k}$ and setting it to zero, we obtain the following equation: 


$$
\sum_{n=1}^{N} \gamma\left(z_{n k}\right)\left\{\frac{\lambda_{2 k}-\lambda_{1 k}}{\lambda_{1 k} \lambda_{2 k}} \times\left(\frac{1}{2}\left(a_{1 n k}^{2}-a_{2 n k}^{2}\right) \sin 2 \theta_{k}-a_{1 n k} a_{2 n k} \cos 2 \theta_{k}\right)\right\}=0
$$

where $\left(a_{1 \mathrm{nk}}, a_{2 \mathrm{nk}}\right)$ is $\left(\mathbf{x}_{n}-\boldsymbol{\mu}_{k}\right)^{T}$. The solution of this equation is given by

$$
\text { if } \sum_{n=1}^{N} \gamma\left(z_{n k}\right)\left(a_{1 n k}^{2}-a_{2 n k}^{2}\right)=0 \Rightarrow \theta_{k}=\frac{\pi}{4} \text { and } \frac{3 \pi}{4}
$$

$$
\text { otherwise } \quad \begin{aligned}
\theta_{k}^{\prime} & =\frac{1}{2} \arctan \left(\frac{2 \sum \gamma\left(z_{n k}\right) a_{1 n k} a_{2 n k}}{\sum \gamma\left(z_{n k}\right)\left(a_{1 n k}^{2}-a_{2 n k}^{2}\right)}\right) \\
\theta_{k} & =\left\{\begin{array}{l}
\theta_{k}^{\prime}+\frac{\pi}{2} \text { and } \theta_{k}^{\prime}+\pi\left(\theta_{k}^{\prime}<0\right) \\
\theta_{k}^{\prime} \text { and } \theta_{k}^{\prime}+\frac{\pi}{2}\left(\theta_{k}^{\prime} \geq 0\right)
\end{array}\right.
\end{aligned}
$$

The two possible solutions represent the local maximum and local minimum. By selecting the one whose second order differential is negative, the solution for the local maximum is obtained and passed on to the next iteration of the EM algorithm.

When it comes to real datasets, animal individuals sometimes move too fast and the solutions to the EM algorithm from the previous frame could become inappropriate as the initial parameters. These 'loss of individual' events are detected by calculating the likelihood function for a mixture component $k$ with the initial parameter values $\mu_{k}$ and $\boldsymbol{\Sigma}_{k}$. If the calculated likelihood is less than a threshold $\alpha$, a round of $\mathrm{K}$-means ++ algorithm is performed by fixing the parameters of all other components, and $\mu_{k}$ and $\boldsymbol{\Sigma}_{k}$ are updated as described earlier. On the other hand, if no 'loss of individual' events are detected, noise reduction is then conducted where any pixel whose likelihood, according to the initial parameters, lies below a threshold $\beta$ is regarded as noise and removed. In the current implementation, $\alpha=\beta=10^{-15}$.

\subsection{Post-processing Step}

\subsubsection{Identity-Swapping Alert Step}

As described earlier, the tracking step preserves the identity of each individual across frames in most cases; however, identity-swapping errors may occur at frames that contain occlusion. This step alerts the user to them. 
A

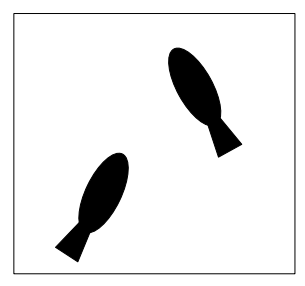

B

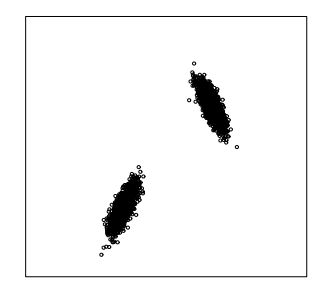

$\mathrm{C}$

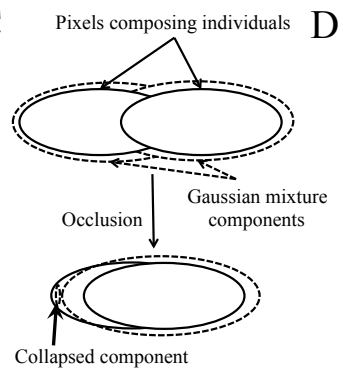

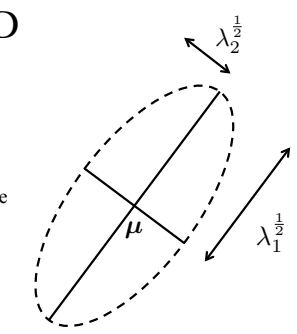

Figure 3: (A) A schematic illustration of a preprocessed image frame. (B) Twodimensional Gaussian mixture representation of the same image frame. (C) A schematic illustration of a case that two Gaussian mixture components overlap and one of component collapses to a single pixel. (D) A schematic illustration of the interpretation of eigenvalues and a covariance matrix of a Gaussian distribution. $\lambda_{i}$ represents the two eigenvalues, while $\mu$ and the ellipse represent the mean value and a constant probability density contour, respectively.

First, for each pixel $\mathbf{x}_{n}$ in each frame, this step finds $k 1, k 2 \in\{k \mid 1 \leq$ $k \leq K\}$ that constitute the largest and second largest values of $\gamma\left(z_{n k}\right)$, i.e., the top two mixture components that best explain $\mathbf{x}_{n}$. A $\gamma\left(z_{n k 1}\right)$ value less than a threshold $a=0.7$ indicates that these components get close in that frame. In this case, a combination of the frame number, $k 1$, and $k 2$ are recorded. A series of successive frames allowing at most one-frame gaps with the same recorded component pair $(k 1, k 2)$ are then grouped into an "incident". Incidents spanning less than a threshold $b=5$ frames are discarded to exclude potential false positives. In addition, the differences between angles $\theta_{k 1}$ and $\theta_{k 2}$ of the two recorded components are calculated for all frames within an incident and, if the minimum difference is larger than a threshold $c=\pi / 6$, that incident is discarded. This is because large angle differences result in large Kullback-Leibler divergences between the mixture components that prevent identity-swapping errors. Finally, the remaining incidents are presented to the user as possible cases of identity-swapping errors.

\subsubsection{Identity-Swapping Correction Step}

Not every identity-swapping error can be corrected completely automatically. This step aims at correcting errors by detecting unnatural sudden changes in directions or speed of each individual's movement.

Given a user-defined value $t_{0}$ (default $t_{0}=10$ ) and an incident beginning 
at frame number $f_{\text {first }}$ and ending at frame number $f_{\text {last }}$, the values of $\mu_{k}$ for the recorded components $k 1$ and $k 2$ at frames $\left(f_{\text {first }}-t_{0}\right), \frac{1}{2}\left(f_{\text {first }}+\right.$ $\left.f_{\text {last }}\right)$, and $\left(f_{\text {last }}+t_{0}\right)$ are extracted. For simplicity, we defined them as $\mu_{k \_ \text {pre }}, \mu_{k \_ \text {mid }}$, and $\mu_{k_{-} \text {post }}$, respectively. To look for sudden changes in directions, the angle formed by $\mu_{k 1 \_ \text {pre }}, \mu_{k 1 \_ \text {mid }}$, and $\mu_{k 1 \_ \text {post }}$ and the angle formed by $\mu_{k 2}$ pre, $\mu_{k 2 \_ \text {mid }}$, and $\mu_{k 2 \text { _post }}$ are examined. If both angles are smaller than a threshold $d=\pi / 2$, the incident is judged as an identityswapping error and corrected by re-swapping the identities mapped to the two components starting from the frames of the incident (Supplementary Fig. 2A). To detect sudden changes in speed, we first define the distances $d_{1 \text { pre }}, d_{1 \text { post }}, d_{2 \text { pre }}$, and $d_{2 \text { post }}$ as $\left|\mu_{k 1 \_p r e}-\mu_{k 1 \_ \text {mid }}\right|,\left|\mu_{k 1 \_ \text {mid }}-\mu_{k 1 \_ \text {post }}\right|$, $\left|\mu_{k 2 \_ \text {pre }}-\mu_{k 2 \_ \text {mid }}\right|$, and $\left|\mu_{k 2 \_ \text {mid }}-\mu_{k 2 \_ \text {post }}\right|$, respectively. Then, if $d_{\text {score }}=$ $\left|d_{1 \text { pre }}-d_{1 \text { post }}\right|+\left|d_{2 \text { pre }}-d_{2 \text { post }}\right|-\left|d_{1 \text { pre }}-d_{2 \text { post }}\right|-\left|d_{2 \text { pre }}-d_{1 \text { post }}\right|$ is greater than a threshold $e=20$ pixels, the incident is judged as an identity-swapping error and corrected in the same manner (Supplementary Fig. 2B).

\subsubsection{Head-Direction Determination Step}

At the tracking step, we introduced $\theta_{k} \in[0, \pi)$, which represents the angle of the major axis of the Gaussian component representing individual $k$. The upper limit was $\pi$ instead of $2 \pi$, because the covariance matrices are diagonal and did not discriminate between the head and tail of an individual. At this step, the head directions of the individuals are explicitly determined and $\theta_{k}$ is updated to be in the range $[0,2 \pi)$.

First, because the head direction of the individual $k$ does not abruptly change between successive image frames, frames are grouped if the differences between their $\theta_{k}$ values are less than $\pi / 4$ (or greater than $3 \pi / 4$ ). Note that, if the individual $k$ does not overlap with any other individuals during the entire video sequence, all frames usually formed a single group. This process is repeated for each individual. For each frame $f$ in the frame group for individual $k$, the velocity $\mathbf{v}_{k}(f)$ is obtained as the difference vector between $\mu_{k}$ at frames $f-t_{0}$ and $f+t_{0}$. At the frame $f_{\max }$ where $\left|\mathbf{v}_{k}\left(f_{\max }\right)\right|$ is maximized, the movement of individual $k$ is assumed to be its head direction. Thus, if the difference between the angle of $\mathbf{v}_{k}\left(f_{\max }\right)$ and $\theta_{k}$ at $f_{\max }$ is greater than $\pi / 2$, to the value of $\theta_{k}$ at $f_{\max }$ is updated to $\theta_{k}+\pi$. Finally, $\pi$ is added to $\theta_{k}$ at any frame so that the differences between $\theta_{k}$ from adjacent frames are always less than $\pi / 4$ (or greater than $7 \pi / 4$ ). 


\section{Results}

\subsection{Application to Medaka Video Sequences}

GroupTracker was applied to five ten-minute video sequences that recorded one, two, four, eight, and sixteen individuals (Supplementary Movies 1-5, respectively). Figure 1B shows the final product of the preprocessing step of a raw image frame shown in Figure 1A (intermediate states are shown in Supplementary Fig. 3). For most frames, the preprocessing step successfully identified pixels that constitute animal shapes. Any noise pixels that remained were removed by the noise reduction algorithm at the tracking step (Supplementary Fig. 4). Figure 1C shows the movement tracks of the mean values of the eight Gaussian components during a one-minute video segment. Supplementary Movies 6-10 contain the tracking results corresponding to Supplementary Movies 1-5, respectively.

The present method requires several user-defined parameters that largely depend on the nature of the video data and the desired applications. Among these parameters, we note that the number of $\mathrm{K}$-means ++ trials $R$ should be set sufficiently large to increase the accuracy of the subsequent EM algorithm. For example, setting $R=1$ resulted in incorrect clusterings for the cases of eight and sixteen medaka individuals. On the other hand, setting $R=100$ yielded 100\% accuracy in every case (Supplementary Fig. 5).

\subsection{Evaluation of Identity-Swapping Errors}

Since it is natural for the user to only manually check the alerted frames for identity-swapping errors, we prioritize sensitivity of the identity-swapping alert and report every frame that might contain the errors. To achieve this, we investigated the sensitivity of the system under various choices of threshold parameters $a, b$, and $c$ (see Material and Methods). Manual inspection of all identity-swapping errors (Supplementary Table 1) confirmed that the sensitivity was $100 \%$ for $a \geq 0.6$ (Supplementary Fig. 6A , $b=0$ and $c=\pi / 2$ are fixed). By definition, the larger $a$ was, the more frames were recorded during the first phase of the identity-swapping alert step (Supplementary Fig. 6B). Nonetheless, the actual number of alerted incidents did not monotonically increase with $a$ and in some instances reached its minimum value around $a=0.7$ (Supplementary Fig. 6C), probably because some incidents were mistakenly divided into smaller ones when the value of $a$ was too small. Then, we fixed $a=0.7$, and optimized the values for $b$ and $c$ so that the $100 \%$ sensitivity is maintained while minimizing the number of incorrectly 
reported incidents. We found that setting $b=5$ frames and $c=\pi / 6$ is appropriate because the minimum length of confirmed incidents was 9 frames and the maximum angle difference of the two individuals in an incident was $19^{\circ}$ (Supplementary Figs. 7A and 7B). With these threshold values, the precision of the identity-swapping alert step was improved from 0.033 to 0.143 while maintaining perfect sensitivity (Supplementary Fig. 7C).

On the other hand, at the identity-swapping correction step, precision becomes fundamental, i.e., false positives should be avoided. We found that setting $d=\pi / 2$ and $e=20$ pixels (see Material and Methods) safely maintains $100 \%$ precision. For incidents that did not contain identity-swapping errors, the angles defined by individual's changes in direction are well above $\pi / 2$ (data not shown), and the maximum $d_{\text {score }}$ was 14 pixels (Supplementary Fig. 7D).

Next, we evaluated the performance of our system regarding the identityswapping errors by calculating the accuracy of the correction step over all image frames where some fish individuals overlap. First, we extracted all image frames in which individuals are confirmed to be overlapped (Supplementary Table 2). In the present dataset, we could manually judge every identity-swapping case without ambiguity. Table 1 summarizes the accuracy of the systems with and without the identity-swapping correction step. Although the overall accuracy was already high (0.88) even without any corrections, utilizing the identity-swapping correction step improved it to 0.92 . These results show that the system accurately preserves individual identities under occlusion.

\section{Tables}

Table 1: Ratios of cases that identities were correctly preserved by the system.

\begin{tabular}{|c|ccccc|}
\hline & $K=2$ & $K=4$ & $K=8$ & $K=16$ & Total \\
\hline Without correction step & $\mathbf{1 . 0 0}$ & 0.72 & $\mathbf{0 . 9 3}$ & 0.88 & 0.88 \\
With correction step & $\mathbf{1 . 0 0}$ & $\mathbf{0 . 8 0}$ & $\mathbf{0 . 9 3}$ & $\mathbf{0 . 9 6}$ & $\mathbf{0 . 9 2}$ \\
\hline
\end{tabular}

The bold figures indicate the better value in each case.

\subsection{Evaluation of Position and Angle Estimation}

Next, we evaluated the accuracy of the estimated positions and angles, i.e., head-to-tail directions of fish individuals, by comparing them with the 
ground truth that was obtained as follows. We selected one frame per five seconds, i.e., 120 frames per one ten-minute video sequence, and manually measured the coordinates of the head, center, and tail of all individuals. The coordinates of the centers were regarded as the ground truth for the positions, and the angles of the difference vectors between the head and tail coordinates were regarded as the ground truth for the angles.

Tables 2 and 3 show the percentile errors of the estimated positions and angles, respectively. In all case, $90 \%$ of the estimation fell within the errors of 3.16 pixels in position and 8.31 degrees in angle, and the number of individuals has little effects on these errors. These results show that the estimated positions and angles agree well with the ground truth and also that this performance scales well with the number of individuals (Visualized in Fig. 4).

Table 2: Percentile errors in the estimation of the positions.

\begin{tabular}{|c|ccccc|}
\hline Percentile & $K=1$ & $K=2$ & $K=4$ & $K=8$ & $K=16$ \\
\hline 25th & 1.0 & 1.0 & 1.0 & 1.0 & 1.0 \\
50 th & 1.0 & 1.0 & 1.41 & 1.41 & 1.41 \\
70th & 2.0 & 2.0 & 2.0 & 2.24 & 2.24 \\
90th & 2.24 & 2.24 & 2.83 & 3.16 & 3.0 \\
\hline
\end{tabular}

(in pixels)

\subsection{Evaluation of Running Time}

We evaluated the speed of the system by comparing its running time with the time required for manual annotations. The computation was performed on an Intel(R) Core(TM) i5-3320M 2.6 GHz CPU with 4 GB of memory. Figure 5 shows the times required for the system, those required for manual annotation, and the relative efficiency. Overall, the system is 250-fold to 1800-fold faster than manual annotation and the efficiency increases with the increasing number of individuals. Even in the case of sixteen individuals, the system required less than 90 minutes to process a ten-minute video sequence, and is thus time-efficient enough for practical uses.

\section{Discussion}

In the present study, we developed a multiple animal tracking system called 'GroupTracker'. Its primary algorithm is based on an adaptation of 


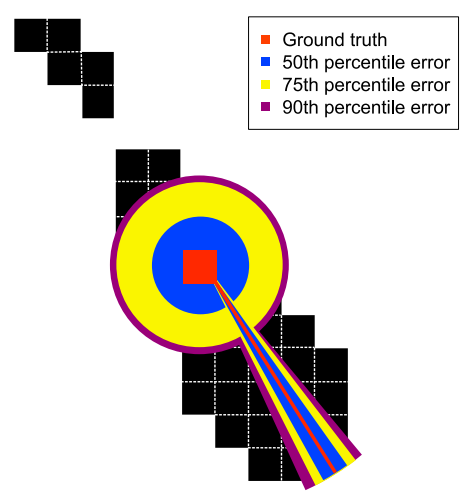

Figure 4: Visualization of percentile errors in the position and angle estimation in the case that the number of individual was one. The black area represents pixels that constituted a medaka shape in an image frame. The red color represents the ground truth, while the blue, yellow, and purple colors represent the ranges of the 50th, 75th, and 90th percentile errors, respectively.

Table 3: Percentile errors in the estimation of the angles.

\begin{tabular}{|c|ccccc|}
\hline Percentile & $K=1$ & $K=2$ & $K=4$ & $K=8$ & $K=16$ \\
\hline 25th & 1.98 & 1.75 & 1.47 & 1.22 & 1.39 \\
50th & 3.68 & 3.35 & 3.37 & 2.84 & 2.79 \\
70th & 5.38 & 5.41 & 5.85 & 5.01 & 4.49 \\
90th & 7.52 & 7.99 & 8.31 & 7.43 & 7.65 \\
\hline
\end{tabular}

(in degrees)

the EM algorithm for Gaussian mixture model with fixed eigenvalues. This enables the system to accurately track individuals under severe occlusion.

We envision four future improvements and utilizations of GroupTracker. The first is speeding up of the system. Although the system already illustrated reasonable time-efficiency, further improvements may be needed, for example, for real-time tracking or tracking of a large flock of animals. This can be achieved by parallelization. In particular, the preprocessing step is clearly parallelizable because the processing of each frame is independent. The tracking and post-processing steps could also be parallelizable if an input video sequence is divided into segments separated by frames where all individuals are clearly identified. The second improvement is implementation of a graphical user interface that allows users to annotate movable areas, visually inspect image frames and alerted incidents, and so on. The third is 


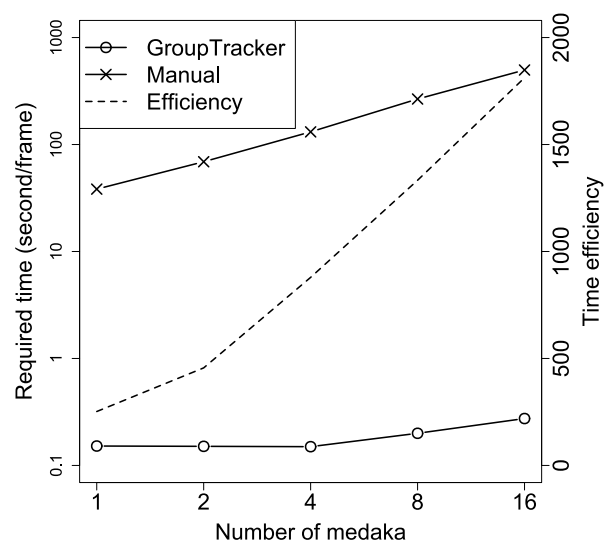

Figure 5: Time required for the system and manual annotation. The x-axis represents the number of medaka individuals. The left y-axis represents time in second per frame in a logarithmic scale (solid lines). The right y-axis represents the time efficiency (dashed line).

the possibility of adopting more-sophisticated machine learning techniques for the detection and correction of identity-swapping errors. Very recently, Pérez-Escudero et al. developed a video-tracking system whose algorithm extracts a characteristic fingerprint from each animal ([29]). Techniques such as the support vector machine have been widely utilized in bioinformatics fields ([8], [15]) and could improve the accuracy, sensitivity, and specificity of tracking. Last but not least, the fourth is the actual utilization of the system to gain novel biological knowledge. We aim at revealing unexplored social network structures ([22], [23]) and behavioral patterns ([7], [4]) behind animal interactions, which would provide insights into the high-order functions of their nervous systems.

\section{Acknowledgements}

This study was supported by Grant-in-Aid for Young Scientists (B) [grant number 23710231] from the Japan Society for the Promotion of Science, the CREST Program from the Japan Science and Technology Agency, and the Canon Foundation. The authors thank Sriswasdi Sira, Haruka Ozaki, and Hirotaka Matsumoto for critically reading the manuscript, and Yasutake Okuda and Satoru Takahashi for technical advice on the tracking system. 


\section{References}

[1] Anken, R. H., Bourrat, F., 1998. Brain atlas of the medakafish: Oryzias latipes. Editions Quae, Versailles.

[2] Arthur, D., Vassilvitskii, S., 2007. k-means++: The advantages of careful seeding. In: Proceedings of the eighteenth annual ACM-SIAM symposium on Discrete algorithms. Society for Industrial and Applied Mathematics, pp. 1027-1035.

[3] Baek, J.-H., Cosman, P., Feng, Z., Silver, J., Schafer, W. R., 2002. Using machine vision to analyze and classify caenorhabditis elegans behavioral phenotypes quantitatively. Journal of neuroscience methods 118 (1), 921.

[4] Berman, G. J., Choi, D. M., Bialek, W., Shaevitz, J. W., 2014. Mapping the stereotyped behaviour of freely moving fruit flies. Journal of The Royal Society Interface 11 (99), 20140672.

[5] Bishop, C. M., Nasrabadi, N. M., 2006. Pattern recognition and machine learning. Vol. 1. Springer, New York.

[6] Branson, K., Robie, A. A., Bender, J., Perona, P., Dickinson, M. H., 2009. High-throughput ethomics in large groups of drosophila. Nature methods 6 (6), 451-457.

[7] Brown, A. E., Yemini, E. I., Grundy, L. J., Jucikas, T., Schafer, W. R., 2013. A dictionary of behavioral motifs reveals clusters of genes affecting caenorhabditis elegans locomotion. Proceedings of the National Academy of Sciences 110 (2), 791-796.

[8] Brown, M. P., Grundy, W. N., Lin, D., Cristianini, N., Sugnet, C. W., Furey, T. S., Ares, M., Haussler, D., 2000. Knowledge-based analysis of microarray gene expression data by using support vector machines. Proceedings of the National Academy of Sciences 97 (1), 262-267.

[9] Dankert, H., Wang, L., Hoopfer, E. D., Anderson, D. J., Perona, P., 2009. Automated monitoring and analysis of social behavior in drosophila. Nature methods 6 (4), 297-303. 
[10] de Chaumont, F., Coura, R. D.-S., Serreau, P., Cressant, A., Chabout, J., Granon, S., Olivo-Marin, J.-C., 2012. Computerized video analysis of social interactions in mice. Nature methods 9 (4), 410-417.

[11] Delcourt, J., Becco, C., Vandewalle, N., Poncin, P., 2009. A video multitracking system for quantification of individual behavior in a large fish shoal: advantages and limits. Behavior Research Methods 41 (1), 228235.

[12] Delcourt, J., Denoël, M., Ylieff, M., Poncin, P., 2013. Video multitracking of fish behaviour: a synthesis and future perspectives. Fish and Fisheries 14 (2), 186-204.

[13] Giancardo, L., Sona, D., Huang, H., Sannino, S., Managó, F., Scheggia, D., Papaleo, F., Murino, V., 2013. Automatic visual tracking and social behaviour analysis with multiple mice. PloS one 8 (9), e74557.

[14] Gonzalez, R. C., Woods, R. E., 2007. Digital Image Processing. Vol. 3. Prentice Hall, Upper Saddle River, NJ.

[15] Hua, S., Sun, Z., 2001. Support vector machine approach for protein subcellular localization prediction. Bioinformatics 17 (8), 721-728.

[16] Kasahara, M., Naruse, K., Sasaki, S., Nakatani, Y., Qu, W., Ahsan, B., Yamada, T., Nagayasu, Y., Doi, K., Kasai, Y., et al., 2007. The medaka draft genome and insights into vertebrate genome evolution. Nature 447 (7145), 714-719.

[17] Kato, S., Nakagawa, T., Ohkawa, M., Muramoto, K., Oyama, O., Watanabe, A., Nakashima, H., Nemoto, T., Sugitani, K., 2004. A computer image processing system for quantification of zebrafish behavior. Journal of neuroscience methods 134 (1), 1-7.

[18] Khan, Z., Balch, T., Dellaert, F., 2005. Mcmc-based particle filtering for tracking a variable number of interacting targets. Pattern Analysis and Machine Intelligence, IEEE Transactions on 27 (11), 1805-1819.

[19] Lloyd, S., 1982. Least squares quantization in pcm. Information Theory, IEEE Transactions on 28 (2), 129-137. 
[20] Magnuson, J. J., 1962. An analysis of aggressive behavior, growth, and competition for food and space in medaka (oryzias latipes (pisces, cyprinodontidae)). Canadian Journal of Zoology 40 (2), 313-363.

[21] Mirat, O., Sternberg, J. R., Severi, K. E., Wyart, C., 2013. Zebrazoom: an automated program for high-throughput behavioral analysis and categorization. Frontiers in neural circuits 7 .

[22] Nagy, M., Ákos, Z., Biro, D., Vicsek, T., 2010. Hierarchical group dynamics in pigeon flocks. Nature 464 (7290), 890-893.

[23] Nagy, M., Vásárhelyi, G., Pettit, B., Roberts-Mariani, I., Vicsek, T., Biro, D., 2013. Context-dependent hierarchies in pigeons. Proceedings of the National Academy of Sciences 110 (32), 13049-13054.

[24] Ofstad, T. A., Zuker, C. S., Reiser, M. B., 2011. Visual place learning in drosophila melanogaster. Nature 474 (7350), 204-207.

[25] Ohayon, S., Avni, O., Taylor, A. L., Perona, P., Roian Egnor, S., 2013. Automated multi-day tracking of marked mice for the analysis of social behaviour. Journal of neuroscience methods 219 (1), 10-19.

[26] Okuyama, T., Yokoi, S., Abe, H., Isoe, Y., Suehiro, Y., Imada, H., Tanaka, M., Kawasaki, T., Yuba, S., Taniguchi, Y., et al., 2014. A neural mechanism underlying mating preferences for familiar individuals in medaka fish. Science 343 (6166), 91-94.

[27] Ono, Y., Uematsu, T., 1957. Mating ethogram in oryzias latipes. Journal of the Faculty of Science Hokkaido University Series VI. Zoology 13 (14), 197-202.

[28] Penagarikano, O., Abrahams, B. S., Herman, E. I., Winden, K. D., Gdalyahu, A., Dong, H., Sonnenblick, L. I., Gruver, R., Almajano, J., Bragin, A., et al., 2011. Absence of cntnap2 leads to epilepsy, neuronal migration abnormalities, and core autism-related deficits. Cell 147 (1), $235-246$.

[29] Pérez-Escudero, A., Vicente-Page, J., Hinz, R. C., Arganda, S., de Polavieja, G. G., 2011. idtracker: Tracking individuals in a group by automatic identification of unmarked animals. Nature Methods 11 (7), $743-748$. 
[30] Ramot, D., Johnson, B. E., Berry Jr, T. L., Carnell, L., Goodman, M. B., 2008. The parallel worm tracker: a platform for measuring average speed and drug-induced paralysis in nematodes. PLoS One 3 (5), e2208.

[31] Silverman, J. L., Yang, M., Lord, C., Crawley, J. N., 2010. Behavioural phenotyping assays for mouse models of autism. Nature Reviews Neuroscience 11 (7), 490-502.

[32] Swierczek, N. A., Giles, A. C., Rankin, C. H., Kerr, R. A., 2011. Highthroughput behavioral analysis in c. elegans. Nature methods 8 (7), 592598.

[33] Tsai, H.-Y., Huang, Y.-W., 2012. Image tracking study on courtship behavior of drosophila. PloS one 7 (4), e34784. 


\section{Graphical Abstract}

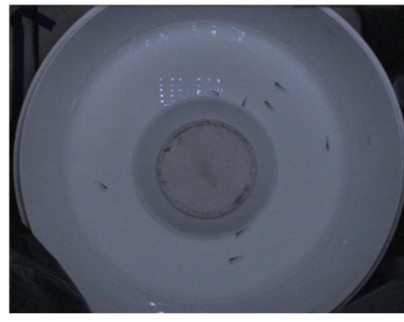

Animal tracking by maximum likelihood estimation of Gaussian mixture model

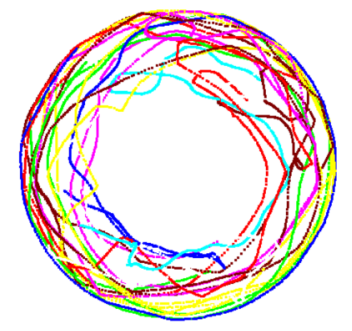

